\title{
Development and validation of molecular markers for characterization of Boehmeria nivea var. nivea and Boehmeria nivea var. tenacissima
}

\author{
Chuan-I Li', Shu-Jiau Chiou', Teng-Soung Tong ${ }^{1,2}$, Cheng-Yu Lee ${ }^{1}$, Lain-Tze Lee ${ }^{1}$, Ching-Ming Cheng ${ }^{3^{*}}$
}

\begin{abstract}
Background: The root of Boehmeria spp (ramie) is a hepatoprotective Chinese herbal medicine. Medicinal properties vary between Boehmeria nivea var. nivea and Boehmeria nivea var. tenacissima, which are local species found in Taiwan. As commercial preparations may use either species, there is a need for a rapid and simple assay to identify variants for quality control.

Methods: Four methods were developed and tested for their applicability in differentiating the two species. These methods were random amplified polymorphic DNA (RAPD); sequence characterized amplified regions (SCAR); single nucleotide polymorphisms (SNP) and cleaved amplified polymorphic sequences (CAPS).

Results: Three RAPD markers were developed that produced unique bands in B. nivea var. tenacissima and B. nivea var. nivea. Based on sequenced RAPD bands, one SCAR marker was developed that produced a single DNA band in B. nivea var. nivea. Two SNP markers differentiated between B. nivea var. nivea and B. nivea var. tenacissima based on single nucleotide substitutions. A pair of CAPS oligonucleotides was developed by amplifying a 0.55-kb DNA fragment that exhibited species-specific digestion patterns with restriction enzymes Alf III and Nde I. Consistent results were obtained with all the four markers on all tested Boehmeria lines.
\end{abstract}

Conclusion: The present study demonstrates the use of the RAPD, SCAR, SNP and CAPS markers for rapid identification of two closely related Boehmeria species.

\section{Background}

The root of Boehmeria species (Urticaceae), namely Boehmeria nivea var. nivea is a hepatoprotective Chinese herbal medicine [1] as well as an antioxidant and anti-inflammatory agent [2]. Sancheti and colleagues have reported its glycosidase and cholinesterase inhibition properties as an anti-diabetic herb to lower blood glucose and cholesterol levels [3]. Compared to B. nivea var. nivea, $B$. nivea var. tenacissima is more hepatoprotective on hepatitis B-induced liver damage [4]. As commercial preparations may consist of one or the other variants, there is a need for rapid and simple assays to identify variants for the purpose of both commercial production and quality control. Whereas today's methods rely primarily on morphological observations,

\footnotetext{
* Correspondence: Ischingming@mail.tcu.edu.tw

${ }^{3}$ Department of Life Sciences, Tzu-Chi University, Hualien 970, Taiwan

Full list of author information is available at the end of the article
}

molecular genetics are a more precise tool, less susceptible to user bias.

Based on four molecular approaches, namely random amplified polymorphic DNA (RAPD), sequence characterized amplified region (SCAR), single nucleotide polymorphism (SNP) and cleaved amplified polymorphic sequence (CAPS), we developed and evaluated a set of authentication techniques for the Boehmeria species and help conserve Chinese medicinal plants in Taiwan.

RAPD is a modified polymerase chain reaction (PCR) technique involving multiple oligonucleotide primers. The resulting amplified DNA markers are random polymorphic segments with band sizes from 100 to $3000 \mathrm{bp}$ depending upon the genomic DNA and the primer. SCARs are DNA fragments amplified by using specific 1530 bp primers, designed from nucleotide sequences established in cloned RAPD fragments. By using longer PCR primers, SCARs have a higher rate of reproducibility than 
RAPDs. SNP analysis is more specific still but requires sequencing to identify the different nucleotides.

CAPS polymorphisms are differences in restriction fragment lengths caused by SNPs that create or abolish restriction endonuclease recognition sites in PCR amplicons. All of these markers are locus-specific with a wide range of applicability in gene mapping and markerassisted selection [5-7]. This article describes the main results of the study.

\section{Methods}

\section{Plant materials}

Eight lines of $B$. nivea var. nivea and $B$. nivea var. tenacissima were collected from various locations of Taiwan and identified by one of the authors (TST), based on the criteria that $B$. nivea var. nivea has a white-grey color with obvious pubescence in their ventral leaf surface and $B$. nivea var. tenacissima has a light green-grey color [8]. Four collections, namely CY1 (Chi-Yi-1), CY2 (Chi-Yi-2), CY3 (Chi-Yi-3) and HCn (Hsin-Chu-n) belong to $B$. nivea var. tenacissima and the other four, namely HCd (Hsin-Chu-d), TC (Tai-Chung), CY (ChiYi) and TARI (Taiwan Agricultural Research Institute) are local variants of $B$. nivea var. nivea.

DNA extraction was performed according to the method described by Arasl et al. [9]. Briefly, $100 \mathrm{mg}$ fresh leaves were ground in liquid nitrogen and transferred to tubes containing $5 \mathrm{~mL}$ CTAB/PVPP extraction buffer which consisted of $0.1 \mathrm{M}$ Tris $\mathrm{HCl}, 1 \mathrm{M} \mathrm{NaCl}, 20 \mathrm{mM}$ EDTA, $1 \%$ hexadecyl trimethylammonium bromide (CTAB; w/ vol) and $1 \%$ polyvinylpolypyrrolidone (PVPP; w/vol). The mixture was incubated at $65^{\circ} \mathrm{C}$ for 20 minutes and extracted with an equal volume of chloroform/isoamylalcohol (24:1). After centrifugation $(8,000 \times g$, Sigma 3-18 K, Germany) for 5 minutes, the supernatant was transferred to a clean tube and precipitated with two volumes of precipitation buffer $(50 \mathrm{mM}$ Tris $\mathrm{HCl}, 4 \mathrm{mM} \mathrm{NaCl}, 10 \mathrm{mM}$ EDTA and $1 \%$ CATB) at $10,000 \times g$ for 20 minutes. The pellet was re-suspended in $350 \mu \mathrm{L} 1.2 \mathrm{M} \mathrm{NaCl}$ and incubated with $10 \mathrm{mg} / \mathrm{mL}$ RNase at $37^{\circ} \mathrm{C}$ for 30 minutes. After extraction with an equal volume of chloroform/isoamylalcohol (24:1), the DNA pellet was re-precipitated with icecold isopropanol, washed with $70 \%$ ethanol, vacuum dried and dissolved in $200 \mu \mathrm{L}$ TE buffer.

\section{RAPD}

RAPD reactions [10] were carried out in a final volume of $25 \mu \mathrm{L}$ containing 1 unit Taq DNA polymerase, 100 $\mu \mathrm{M}$ dNTP mixture, $10 \mathrm{mM}$ Tris $\mathrm{HCl}, 1.5 \mathrm{mM} \mathrm{MgCl}_{2}$, 1.0 $\mu \mathrm{M}$ primer and 10-20 ng template DNA. Amplification was performed in a PCR machine (Thermocycler 2100 , PerkinElmer, USA) at $94^{\circ} \mathrm{C}$ for two minutes followed by 40 cycles of 30 seconds at $94^{\circ} \mathrm{C}, 40$ seconds at $36^{\circ} \mathrm{C}, 45$ seconds at $72^{\circ} \mathrm{C}$ and a final stage of five minutes at $72^{\circ} \mathrm{C}$. The amplification products were maintained at $4{ }^{\circ} \mathrm{C}$ and resolved in $1.5 \%$ agarose gel followed by ethidium bromide staining and visualization with UV light for photography. The amplified DNA fragments, RP-S343-1.1, RP-S343-0.9 and RP-S62-0.6 were used for oligonucleotide design (Table 1). To avoid sequences that would produce internal secondary structures, we checked primers with Oligo 6 software (National Sciences, USA)

\section{SCAR}

Three pairs of oligonucleotides were used in the SCAR assays [11], namely forward oligonucleotide SR-S343-F1 and the three reverse oligonucleotides SR-S343-R1, SRS343-R2 and SR-S343-R3 (Table 1). The SCAR reaction was performed with an initial denaturation step at $95^{\circ} \mathrm{C}$ for five minutes, followed by 35 cycles of $94^{\circ} \mathrm{C}$ for two minutes, $60^{\circ} \mathrm{C}$ for one minute, $72^{\circ} \mathrm{C}$ for one minute and a 10-minute final extension at $72^{\circ} \mathrm{C}$.

PCR fragments were cloned with TA cloning technology using pGEM-T-Easy vectors (Promega, USA) and used to transform the Escherichia coli strain XL-2 Blue (Stratagene, USA). DNA sequence analysis was carried out with the BLAST sequence analysis programs at the National Center for Biotechnology Information (NCBI) [12]. Alignments were edited with the online ClustalW program from DNA Data Bank of Japan [13].

\section{SNP}

A sequence from a RAPD DNA fragment, namely RPS62-0.6, was chosen for SNP detection. The procedures were performed according to the manufacturer's instructions [14] with one modification, i.e. the mixture solution was diluted 1:8 with magnesium buffer $(400 \mathrm{mM}$ Tris $\mathrm{pH} 9$ and $10 \mathrm{mM} \mathrm{MgCl}_{2}$ ). Each reaction contained $0.5 \mu \mathrm{L}$ of the SNaPshot ${ }^{\mathrm{tw}}$ Multiplex Ready Reaction Mix (Applied Biosystems, USA), $2.0 \mu \mathrm{L}$ of PCR product, 1.0 $\mu \mathrm{L}$ of extension primers and water up to $10 \mu \mathrm{L}$. Thermal cycling and post-extension were run on an $A B I$ Prism 3100 Genetic Analyzer (Applied Biosystems, USA).

\section{CAPS}

CAPS analyses were performed according to published methods [15]. The RP-S62-0.6 DNA fragment was amplified from the eight Boehmeria lines with primers CP-S62-f and CP-S62-r. DNA fragments were digested with restriction enzymes (i.e. Afl III, Bsr FI, Msp I, Drd I and Nde I) and separated on a $1.5 \%$ agarose gel for polymorphism detection.

\section{Quality control}

A mixture of DNA was used to identify the basis of all the markers for quality control. Samples contained DNA 
Table 1 Primers used for marker analysis

\begin{tabular}{|c|c|c|c|c|c|}
\hline Technique & $\begin{array}{l}\text { Annealing } \\
\text { temperature }\left({ }^{\circ} \mathrm{C}\right)\end{array}$ & $\begin{array}{l}\text { Name of the } \\
\text { primer }\end{array}$ & Sequence & $\begin{array}{l}\text { Number of } \\
\text { polymorphic bands }\end{array}$ & $\begin{array}{l}\text { Marker } \\
\text { length } \\
\text { (kb) }\end{array}$ \\
\hline \multirow[t]{2}{*}{ RAPD } & $35-40$ & S62 & GTGAGGCGTC & 1 & 0.6 \\
\hline & & S343 & TCGTGCGGGT & 2 & $0.9,1.1$ \\
\hline \multirow[t]{4}{*}{ SCAR } & $55-60$ & SR-S343-F1 & СTCTTGAGCAATCCAAATGTTTGTTATCA & & \\
\hline & & SR-S343-R1 & CATAAATCACTTTATAACATAACGAGCTCGTATT & 1 & 1.03 \\
\hline & & SR-S343-R2 & CGCGACAGAGGGGTTTTCTTTCTATTA & 1 & 0.95 \\
\hline & & SR-S343-R3 & AGACGCCTCACTTTGATAGACATGAGTTTA & 1 & 0.89 \\
\hline \multirow[t]{2}{*}{ SNP } & $50-60$ & Sn-S62-a & CGACAGTAAACATAAAAACCG & 1 & $1^{*}$ \\
\hline & & Sn-S62-b & CTGTTACCATTGGCTCTITACC & & \\
\hline \multirow[t]{2}{*}{ CAPS } & $60-67$ & CP-S62-f & TCGTGCGGGTCATAGTACCCCGAGACAAGAGGCCAAAA & 2 & $\begin{array}{l}0.25,0.3 \\
(\text { Afl III) }\end{array}$ \\
\hline & & CP-S62-r & TGTAATACGAAAGTITAAGTCTCTITTCTTAGTC & & $\begin{array}{l}0.2,0.35 \\
(\text { Drd I) }\end{array}$ \\
\hline
\end{tabular}

* Only one base with different colors. The fluorescent dyes are assigned to the individual ddNTPs. Each ddNTP is labeled with a different color, ddATP green, ddCTP blue, ddGTP black, and ddTTP red.

from both $B$. nivea var. nivea and $B$. nivea var. tenacissima in the ratios of $9: 1,8: 2,7: 3,6: 4,5: 5,4: 6,3: 7,2: 8$ and $1: 9$ respectively.

\section{Results and Discussion \\ RAPD markers for quick screening}

Out of a set of 100 RAPD primers, two primers, namely S343 and S62, produced clear reproducible unique patterns easily distinguishable from one another (Figure 1A and Figure $1 \mathrm{~B}$ ) and were selected for further investigation. The RAPD marker S343 produced two polymorphic bands of $1.1 \mathrm{~kb}$ and $0.9 \mathrm{~kb}$ unique to the $B$. nivea var. nivea and $B$. nivea var. tenacissima species, respectively, while the S62 RAPD marker produced one polymorphic band of $0.6 \mathrm{~kb}$ in $B$. nivea var. tenacissima but none in $B$. nivea var. nivea. The unique bands were amplified, cloned and sequenced. No significantly related genes were found in the GenBank database.

RAPD analysis is fast and economical [16] as long as suitable primers are available. In the present study, only two primers out of 100 species-specific patterns were easily visualized in electrophoresis.

\section{Conversion of RAPD into SCAR and SNP}

The SCAR reaction generated a unique band with $B$. nivea var. nivea DNA but no unique band with $B$. nivea var. tenacissima (Figure 2). SCAR analysis with primers developed from cloned variant-specific RAPD bands is highly specific. We identified three SCAR profiles with single bands easily visualized on agarose gels (Figure 2). As SCAR primers are sequence-specific, this method is less complex and more sensitive than RAPD. SCAR appears to be the method of choice for the characterization of mixtures of both Boehmeria variants in commercial herbal preparations.
The 0.6-kb RAPD fragment that generated from primer S62 was sequenced to identify species-specific SNPs. By using the SNaPshot as identification tools, the primers Sn-S62-f and Sn-S62-r indicated single nucleotide replacements of guanine to adenine and cytosine to guanine in B. nivea var. nivea and B. nivea var. tenacissima, respectively (Figure 3 ).

Only a fraction of the recommended SNaPshot mixtures was used in this study because the quality of SNP detection can be maintained using only one eighth of the recommended amounts of the reagents.

\section{Conversion of SNP sites into CAPS}

Sequence analysis of the S62-amplified fragments revealed several point mutations between the two Boehmeria species. Two of these mutation sites were selected for the CAPS assay, providing a total amount of five possible altered restriction enzyme sites (i.e. $A f l$ III, Bsr FI, Msp I, Drd I and Nde I) between B. nivea var. nivea and $B$. nivea var. tenacissima (Figure 4). The modification of the sequencing protocol did not reduce the accuracy of the sequencing reaction required to identify species-specific SNPs. By digestion of the 0.55 kb RAPD fragments, i.e. Ca-Afl-0.55 and Ca-Nde-0.55 that amplified from the S62 primer and cut with $A f l$ III and Nde I, we produced DNA fragments with predicted sizes of $0.30 \mathrm{~kb}$ and $0.25 \mathrm{~kb}$ for CAPS markers Ca-Afl0.30 and $\mathrm{Ca}-\mathrm{Afl}-0.25$, and $0.35 \mathrm{~kb}$ and $0.20 \mathrm{~kb}$ for CAPS markers Ca-Drd-0.35 and Ca-Drd-0.20 that could be easily visualized. The species-specific patterns of CAPS markers digested with restriction enzyme $A f l$ III and Nde I are shown in Figure 5. The CAPS markers clearly distinguished between the two Boehmeria species. 


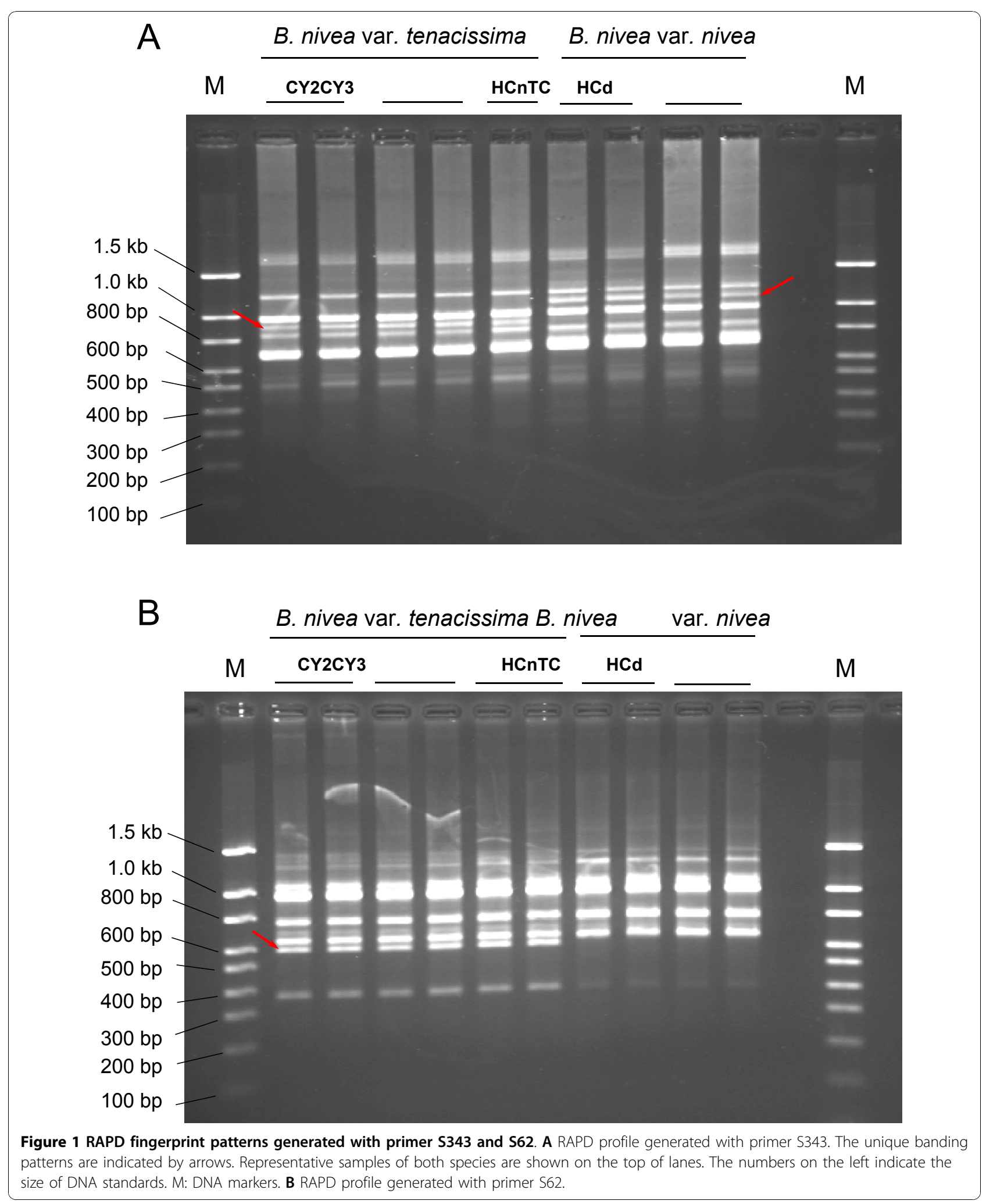




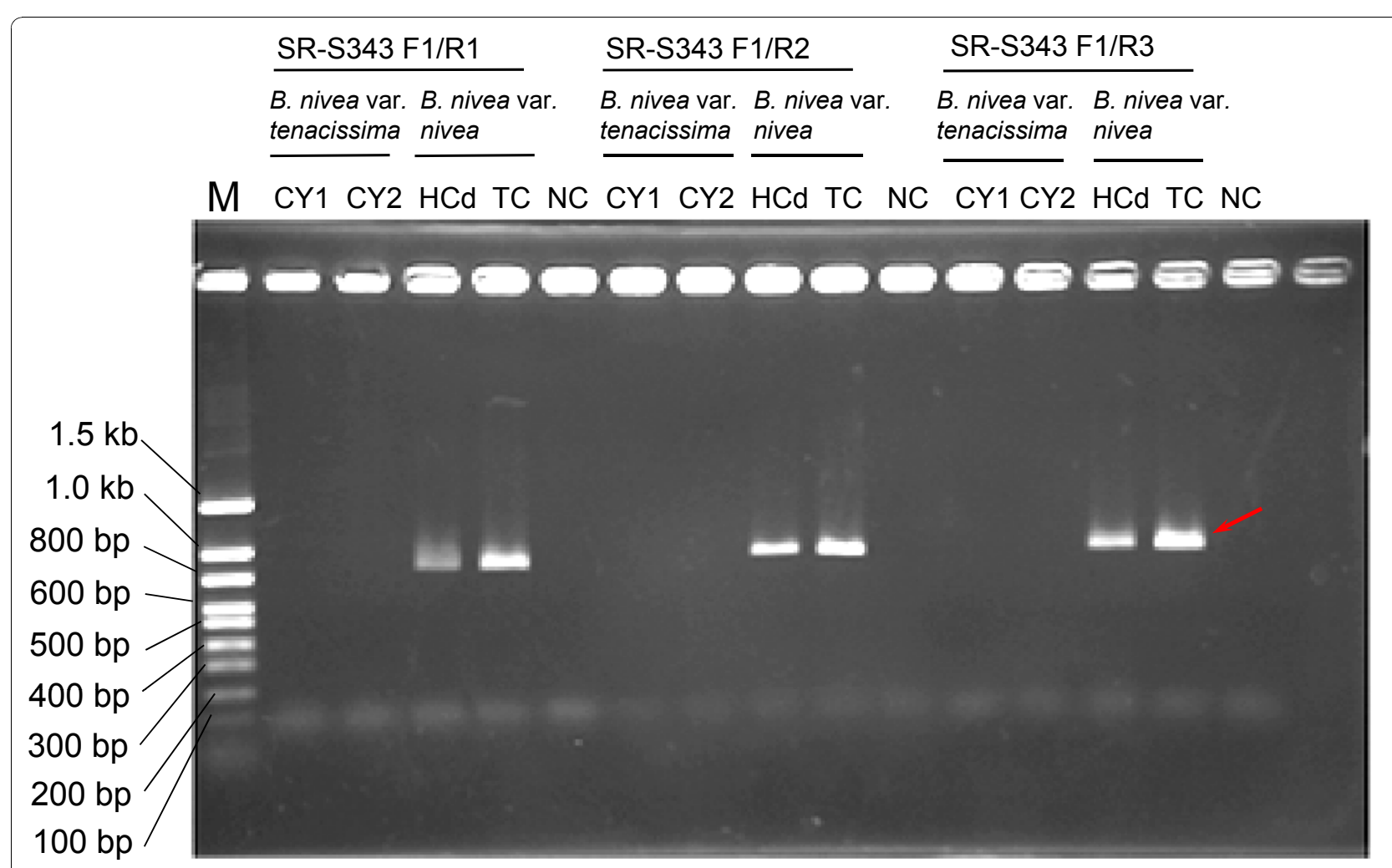

Figure 2 SCAR band patterns of B. nivea var. tenacissima and B. nivea var. nivea. SCAR profiles of Boehmeria DNA fragments generated with primers SR-S343-F1, SR-S343-R1, SR-S343-R2 and SR-S343-R3. Representative samples of both species are shown on the top of lanes. The numbers on the left indicate the size of DNA standards. M: DNA markers; NC: negative control.

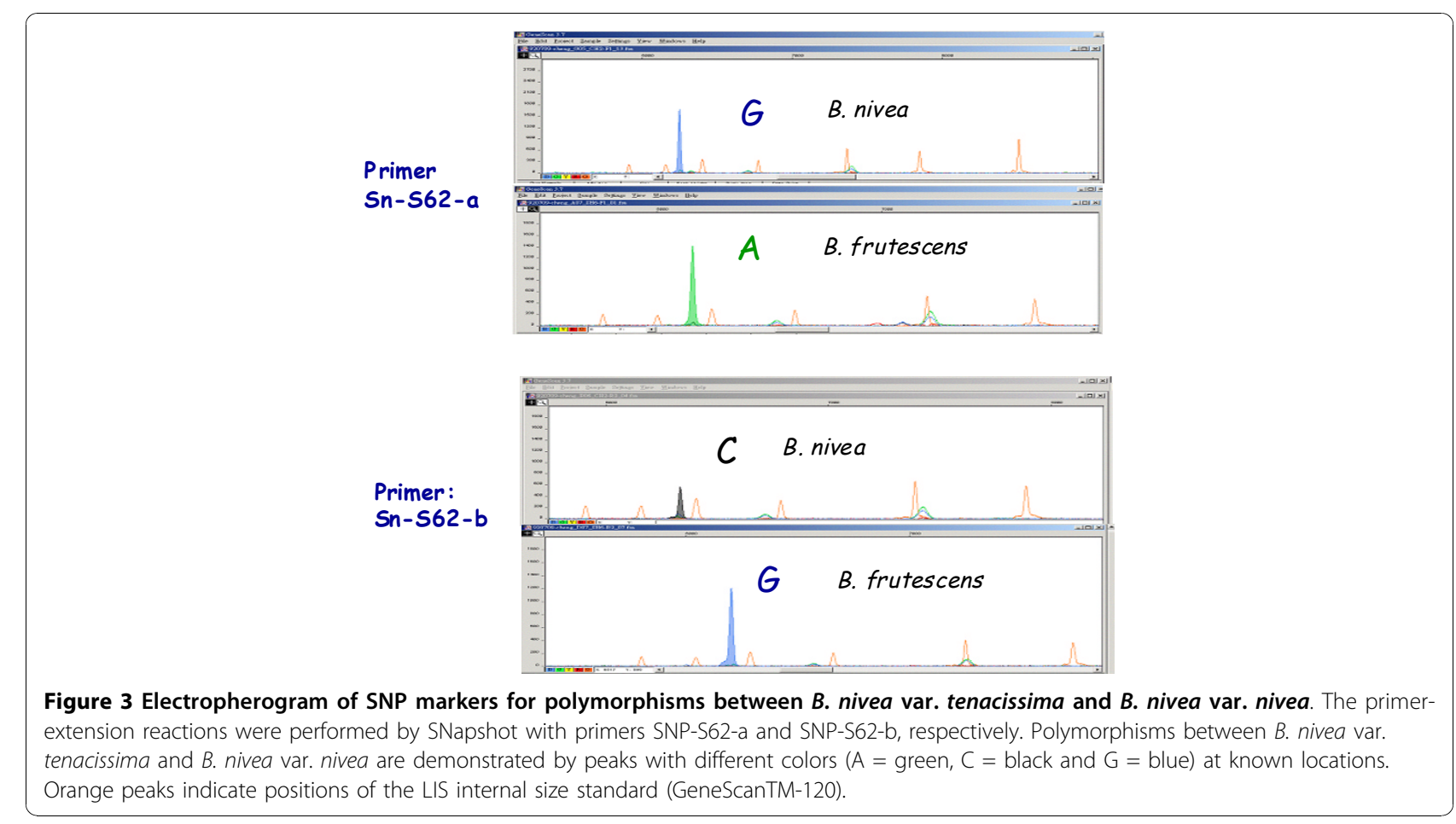


$\operatorname{BrsF} I(A / C C G G C)$

$\operatorname{Msp} I(C / C G G)$

$\operatorname{Drd}$ I (GACNNNN/NNGTC)

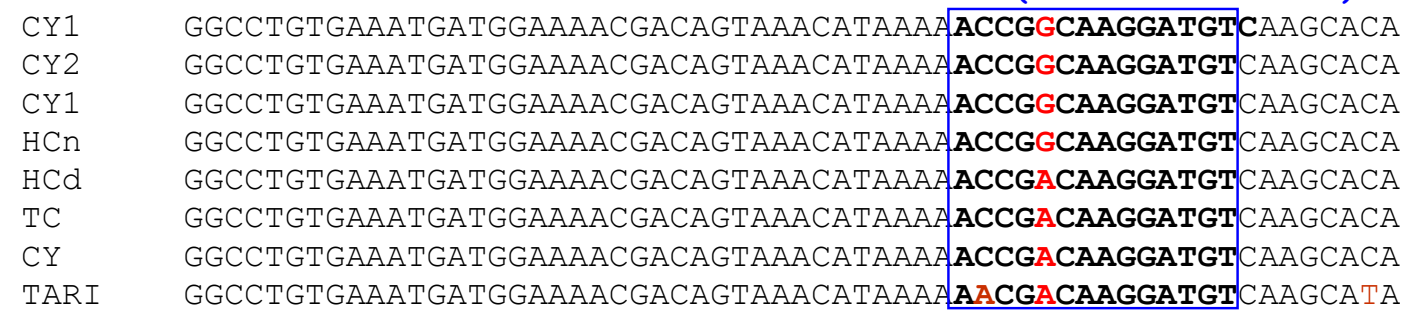

CY1 GATGAAGTGAGGCTTTGCTGTACTGTTATAATGTAGCCGTCTCTTGATCAGTGATCAAAT CY2 GATGAAGTGAGGCTTTGCTGTACTGTTATAATGTAGCCGTCTCTTGATCAGTGATCAAAT CY3 $\mathrm{HCn}$ $\mathrm{HCd}$ $\mathrm{TC}$ CY TARI GATGAAGTGAGGCTTTGCTGTACTGTTATAATGTAACCGTCTCTTGATCAGTGATCAAAT GATGAAGTGAGGCTTTGCTGTACTGTTATAATGTAACCGTCTCTTGATCAGTGATCAAAT GATGAAGTGAGGCTTTGCTGTACTGTTACAATGTAACCGTCTCTTGATCAGTGATCACAT GATGAAGTGAGGCTTTGCTGTACTGTTATAATGTAACCGTCTCTTGATCAGTGATCACAT GATGAAGTGAGGCTTTGCTGTACTGTTATAATGTAACCGTCTCTTGATCAGTGATCACAT GATGAAGTGAGGCTTTTCTGTACTGTTATAATGTAACCGTCTCTTGATAAGTGATCCCAT

Afl III (A/CATGT)
NdeI (CA/TATG)
CY1
CY2

Figure 4 Primer design for CAPS markers. A 0.55-kb fragment was amplified from the eight Boehmeria lines with primers CP-S62- $f\left(5^{\prime}\right.$ CTGAGGCGGGAGCTAGGATTTCAACTAA-3') and CP-S62-r (5'-GGGGGAAGTAGTGCAGCACATGAATATA-3'). For the CAPS analysis, the restriction enzymes that generate polymorphic patterns were found by the dCAPS Finder 2.0 software (http://helix.wustl.edu/dcaps/dcaps.html). Five restriction enzymes (i.e. Afl III, Bsr Fl, Msp I, Drd I and Nde) were used.

\section{Potential applications in quality control}

To test the applicability of the markers, we selected CAPS analysis to trace the identification of components in preparations containing mixtures of DNA from both Boehmeria species. The results (Figure 6) indicated a shift in the electrophoretic patterns corresponding to the increasing and decreasing amounts of DNA from either species. The results confirmed the association of the Ca-Drd-0.55, Ca-Afl-0.30 and Ca-Afl-0.25 markers to $B$. nivea var. nivea and the Ca-Afl-0.55, Ca-Drd-0.35 and Ca-Drd-0.20 CAPS markers to $B$. nivea var. tenacissima.

Based on known SNPs, CAPS is a suitable strategy for the analysis of mixed samples. The present study identified the Boehmeria species in a sample and characterized the relative amounts of one species vs. another in a single sample. While CAPS may be used as a rapid analysis kit for Boehmeria-based preparation compared with the time-consuming RFLP analysis, however it is rather laborious and requires the use of restriction enzymes.

\section{Conclusion}

The present study demonstrates the usefulness of the RAPD, SCAR, SNP and CAPS markers for rapid identification of variants between two closely related Boehmeria species. In particular, CAPS would be a suitable strategy for the analysis of mixed samples.

\section{Abbreviations}

RAPD: random amplified polymorphic DNA; SCARs: sequence characterized amplified regions; SNP: single nucleotide polymorphism; CAPS: cleaved amplified polymorphic sequences; CY1: Chi-Yi-1; CY2: Chi-Yi-2; CY3: Chi-Yi-3; HCn: Hsin-Chu-n; HCd: Hsin-Chu-d; TC: Tai-Chung; CY: Chi-Yi; TARI: Taiwan 


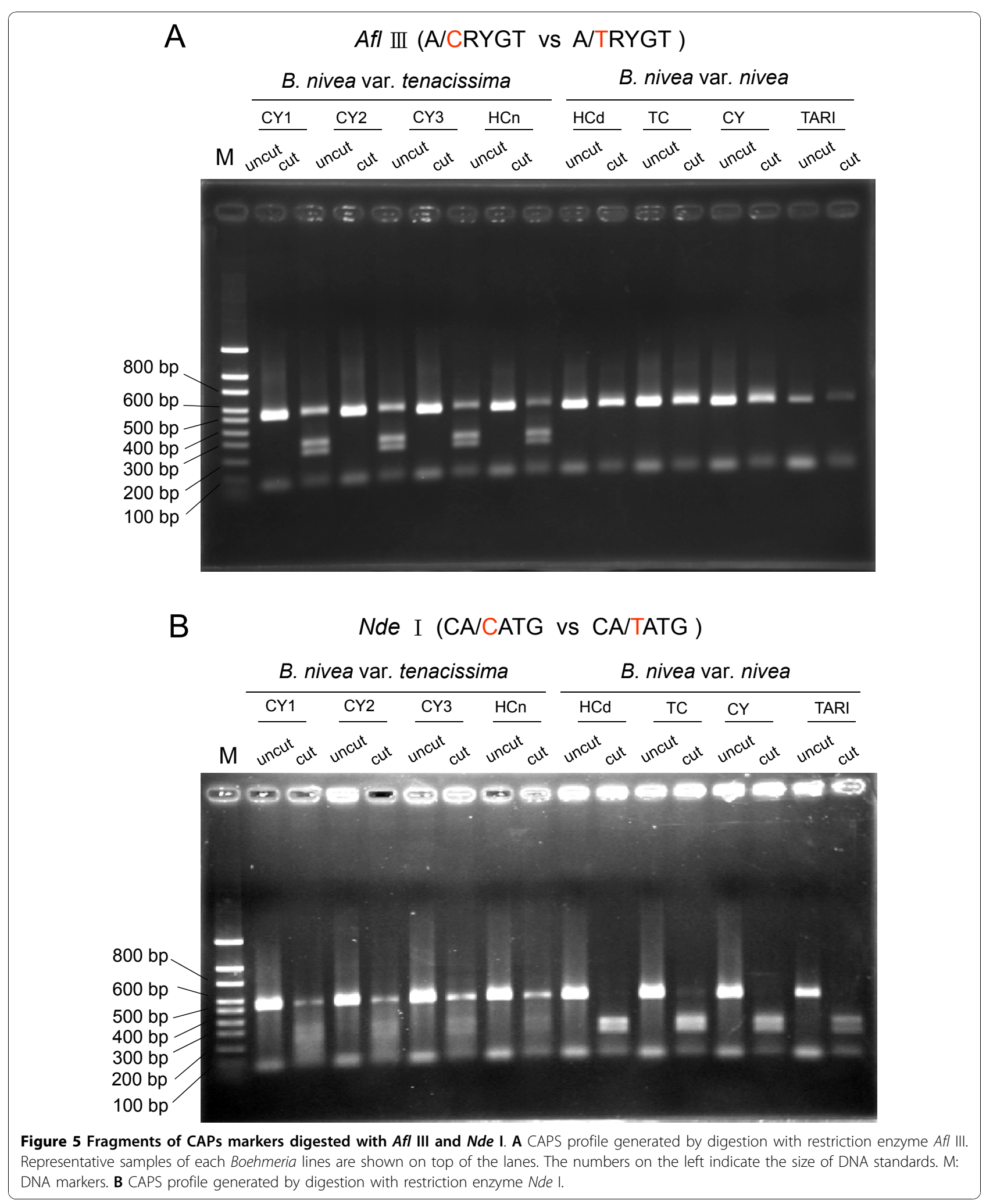




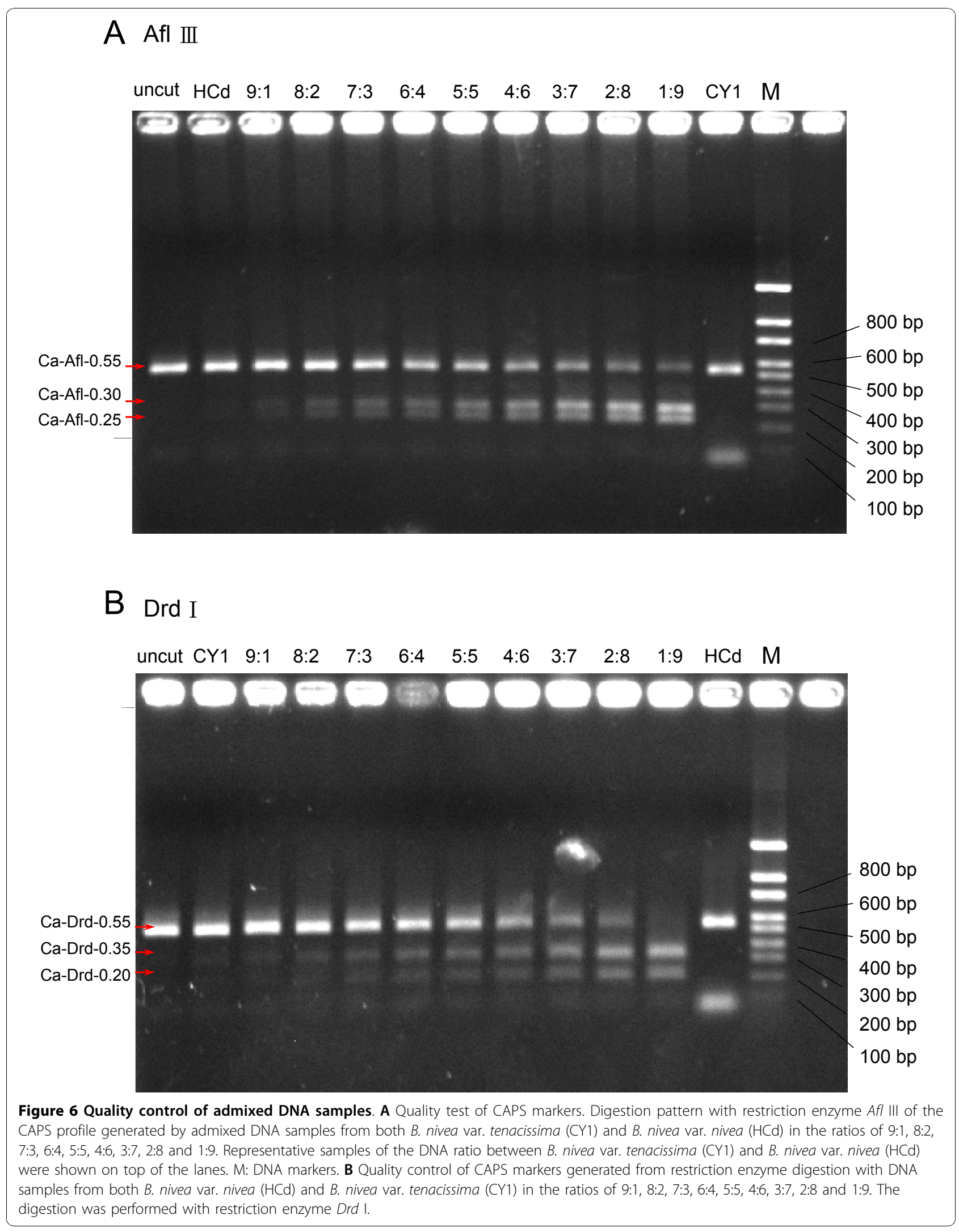


Agricultural Research Institute; CTAB: hexadecyl trimethylammonium bromide; PVPP: polyvinylpolypyrrolidone; PCR: polymerase chain reaction

\section{Acknowledgements}

The authors thank Dr. Pelle Stolt for editorial comments on the manuscript This work was financially support by the Council of Agriculture, the Executive Yuan (93N705-921-G) through NSTP.AB and the Industrial Technology Research Institute (93-EC-17-A-20-R7-0318), Taiwan.

\section{Author details}

'Biomedical Technology and Device Research Laboratories, Industrial Technology Research Institute, Hsinchu 300, Taiwan. ${ }^{2}$ Graduate Institute of Chinese Pharmaceutical Science, China Medical University, Taichung 404, Taiwan. ${ }^{3}$ Department of Life Sciences, Tzu-Chi University, Hualien 970, Taiwan.

\section{Authors' contributions}

CMC conceived and designed the study. CIL performed the laboratory work and data acquisition. SJC interpreted the data. TST collected and authenticated the plant samples. CYL drafted the manuscript. LTL finalized the manuscript. All authors read and approved the final version of the manuscript.

\section{Competing interests}

The authors declare that they have no competing interests.

Received: 15 May 2010 Accepted: 29 November 2010

Published: 29 November 2010

\section{References}

1. Huang KL, Lai YK, Lin CC, Chang JM: Involvement of GRP78 in inhibition of HBV secretion by Boehmeria nivea extract in human HepG2 2.2.15 cells. J Viral Hepati 2009, 16:367-375.

2. Lin CC, Yen MH, Lo TS, Lin JM: Evaluation of the hepatoprotective and antioxidant activity of Boehmeria nivea var. nivea and B. nivea var. tenacissima. J Ethnopharmacol 1998, 60:9-17.

3. Sancheti S, Sancheti S, Seo S: Evaluation of antiglycosidase and anticholinesterase activities of Boehmeria nivea. Pak J Pharm Sci 2010, 23:236-240.

4. Chang J, Huang K: Complementary and alternative therapies in the treatment of chronic hepatitis B. Hep B Annual 2007, 4:72-106.

5. Karp A, Edwards KJ, Bruford M, Funk S, Vosman B, Morgante M, Seberg O, Kremer A, Boursot P, Arctander P, Tautz D, Hewitt GM: Molecular technologies for biodiversity evaluation: opportunities and challenges. Nat Biotech 1997, 15:625-628.

6. Brown JKM: The choice of molecular marker methods for population genetic studies of plant pathogens. New Phytologist 1996, 133:183-195.

7. Parker PG, Snow AA, Schug MD, Booton GC, Fuerst PA: What molecules can tell us about populations: choosing and using a molecular marker. Ecology 1998, 79:361-382

8. Chen CJ, Lin Q, Friis I, Wilmot-Dear CM, Monro AK: Urticaceae. Flora China 2003, 5:76-189.

9. Arasl S, Duran A, Yenilmez G: Isolation of DNA for RAPD analysis from dry leaf material of some Hesperis L. specimens. Plant Mol Bio Rep 2003, 21:461a-461f.

10. Rafalski JA, Tingey SV: Genetic diagnostics in plant breeding: RAPDs, microsatellites and machines. Trends Genet 1993, 9:275-280.

11. Dianese EC, Fonseca1 MEN, Goldbach R, Kormelink R, Inoue-Nagata1 AK, Resende RO, Boiteux LS: Development of a locus-specific, co-dominant SCAR marker for assisted-selection of the Sw-5 (Tospovirus resistance) gene cluster in a wide range of tomato accessions. Mol Breed 2010, 25:133-142.

12. Altschut SF, Gish W, Miller W, Myers EW, Lipman DJ: Basic local alignment search tool. J Mol Boil 1990, 215:403-410.

13. DNA Data Bank of Japan. [http://clustalw.ddbj.nig.ac.jp/top-e.html].

14. Terwilliger JD, Haghighi F, Hiekkalinna TS, Goring HH: A biased assessment of the use of SNPs in human complex traits. Curr Opin Genet Dev 2002, 12:726-734.

15. Cheng CM, Palloix A, Lefebvre V: Isolation, mapping and characterization of allelic polymorphism of Chi-PM1, a class III chitinase of Capsicum annuum L. Plant Sci 2002, 163:481-489.
16. Hadrys H, Balick M, Schierwater B: Applications of random amplified polymorphic DNA (RAPD) in molecular ecology. Mol Ecol 1992, 1:55-63.

doi:10.1186/1749-8546-5-40

Cite this article as: Li et al:: Development and validation of molecular markers for characterization of Boehmeria nivea var. nivea and Boehmeria nivea var. tenacissima. Chinese Medicine 2010 5:40.

\section{Submit your next manuscript to BioMed Central and take full advantage of:}

- Convenient online submission

- Thorough peer review

- No space constraints or color figure charges

- Immediate publication on acceptance

- Inclusion in PubMed, CAS, Scopus and Google Scholar

- Research which is freely available for redistribution

Submit your manuscript at www.biomedcentral.com/submit
C Biomed Central 